\title{
Effect of Seminal Centrifugation with lodixanol on the Sperm Quality of Goat
}

\section{Semen}

\author{
Sonia Galian*, Begoña Peinado, Angel Poto, and Laura Almela \\ Instituto Murciano de Investigación y Desarrollo Agrario y Alimentario (IMIDA), Equipo de mejora genética animal, La Alberca, Murcia, Spain
}

*Corresponding author: Sonia Galian, Instituto Murciano de Investigación y Desarrollo Agrario y Alimentario (IMIDA), Equipo de mejora genética animal, La Alberca, Murcia, Spain, E-mail: sgalianarnaldos@gmail.com

Received: 27 Jan, 2020 | Accepted: 24 Feb, 2020 | Published: 28 Feb, 2020

Citation: Galian S, Peinado B, Poto A, Almela L (2020) Effect of Seminal Centrifugation with lodixanol on the Sperm Quality of Goat Semen. J Anim Sci Res 4(1): dx.doi.org/10.16966/2576-6457.135

Copyright: ( $) 2020$ Galian S, et al. This is an open-access article distributed under the terms of the Creative Commons Attribution License, which permits unrestricted use, distribution, and reproduction in any medium, provided the original author and source are credited.

\begin{abstract}
Goat semen cryopreservation techniques facilitate the reproductive management of the herd and allow preserving the fertilizing capacity of the spermatozoa for long periods of time. However, they require centrifugation techniques for the removal of the seminal plasma. This centrifugation can mechanically damage the spermatozoon membrane, especially if it is performed at high gravitational forces and for a long time. In order to avoid this mechanical damage, the centrifugation of goat semen is studied with lodixanol (Cushion ${ }^{\circledR}$ Fluid), a compound that acts as a "cushion", avoiding the strong packing of the spermatozoa at the bottom of the centrifugation tube. Seminal samples obtained from Murciano-Granadina goats were divided into 2 tubes, with and without lodixanol, centrifuged at $800 \mathrm{~g}$ for 20 minutes, the supernatant and quilting medium were removed and resuspended in Thilmant medium and refrigerated at $5^{\circ} \mathrm{C}$ for 24 hours. After that time, the quality of movement and \% of motile sperm, the vitality with eosin-nigrosine staining and the \% of morphoanomalies of each type of sample were evaluated. After analyzing the results statistically, we obtained that the post-centrifugation seminal quality does not vary significantly with both treatments, but the lodixanol allows in the goat semen a more complete and less aggressive resuspension and homogenization of the spermatozoa after centrifugation, which would translate into a greater recovery of undamaged spermatozoa.
\end{abstract}

Keywords: Goat; Sperm quality; Refrigerated semen; Seminal centrifugation; lodixanol

\section{Introduction}

Currently, refrigeration and seminal freezing techniques are necessary to facilitate assisted reproduction processes such as artificial insemination in domestic species, as they allow the fertilizing capacity of the spermatozoa to be preserved for longer than fresh semen. This conservation is essential when semen must be transferred from its place of collection to other farms in order to disseminate the genetic material of breeding males. It also facilitates the dissemination of genetic material in situations where the movement of live animals is prohibited or limited, or in the case of frozen semen, it allows the existence of germplasm banks, in which thousands of seminal doses are stored, some even from already deceased animals, which increases genetic diversity and the number of available genetic specimens. Despite the advantages of seminal cryopreservation, we must not forget that it requires a series of guidelines in manipulation that can affect sperm quality and fertilizing capacity. Among these guidelines is centrifugation, used to remove seminal plasma, which is known as seminal lavage. The elimination of seminal plasma becomes necessary when using diluents containing egg yolk, because an interaction occurs between the phospholipase secreted by the bulbourethral glands (present in seminal plasma) that catalyzes the hydrolysis of lipids in the egg yolk to fatty acids and lysophospholipids that are toxic to sperm, even at very low temperatures [1,2]. Centrifugation is therefore a way of eliminating this toxicity, in addition to obtaining a greater number of sperm from the ejaculate for subsequent dilution in the freezing medium [3]. There is no fully accepted explanation of how the centrifugation process damages sperm, but the hypothesis of the adverse effect on the sperm cell of the excessive formation of Reactive Oxygen Species (ROS) that would occur during centrifugation $[4,5]$ and also the direct mechanical damage caused to the sperm membrane [6] are considered. Precisely to mitigate this mechanical damage to the membranes, centrifugation is studied with Iodixanol, a high molecular weight colloid (non-ionic iodine compound) that would protect the sperm from possible damage caused by the high centrifugal force. This Iodixanol medium is denser than the semen and diluent used, so it is deposited at the bottom of the centrifuge tube as a "cushion"; and would act to prevent sperm damage caused by being strongly packed against the hard surface of the tube bottom. This medium would provide a mattress effect to the sperm, which makes it deposit in a dense fluid, but without allowing it to reach the bottom of the tube. It is designed in equine semen to allow longer centrifugations at faster speeds, resulting in higher rates of recovery of undamaged sperm [7]. 
The objective of this work was to verify if there are differences in the seminal quality of Murciano-Granadina goats in samples centrifuged with Iodixanol after being refrigerated at $5^{\circ} \mathrm{C}$ for 24 hours, with respect to those centrifuged without using this compound.

\section{Material and Methods}

To carry out this study, 5 males of the Murciano-Granadina (MG) breed were used. Three of them were adults at the start of the trial (3 to 5 years old), the other two were young males (7 to 11 months old at the start of the study). Seminal quality between adult and young males varied substantially, but what was of interest for this work were the differences between the seminal quality of semen treated with Iodixanol and without it, not the variability between specimens. Even so, the starting qualities of the different samples were taken into account to assess whether Iodixanol had different effects if used with poor quality samples than if used with higher quality seminal samples.

The semen was obtained by artificial vagina for goats and was collected in a glass tube with $1 \mathrm{ml}$ of Krebs-Ringer-Phosphate diluents [8] tempered at $37^{\circ} \mathrm{C}$. The volume of pure semen obtained was measured and diluted to $10 \mathrm{ml}$ with the same diluent to be able to visualize the sperm individually. A $20 \mu \mathrm{l}$ drop of the dilution was deposited on a slide tempered to $37^{\circ} \mathrm{C}$ and viewed at $100 \mathrm{x}$ magnifications on an optical microscope. Individual motility was determined (individual movement quality, subjectively evaluated from 0 to 5 , where 0 corresponds to all immobile spermatozoa and 5 with very rapid progressive spermatozoa movement, table 1) [9], the percentage of motile spermatozoa (subjectively assigning the percentage of spermatozoa that move in the microdrop with respect to the total). The sperm concentration was determined using a spectrophotometer (SpermaCue ${ }^{\star}$ ). An eosin-nigrosine stain [10] was performed, mixing $30 \mu \mathrm{l}$ of sample and $30 \mu \mathrm{l}$ of stain that allowed us to know at 400 magnifications the percentage of viable spermatozoa (they are white, not stained) that appeared after counting 200 sperm cells in different fields. With this same preparation we measure the percentage of morphoanomalies [10] of each sample before it is refrigerated.

The seminal samples were allowed to cool to room temperature (about $23^{\circ} \mathrm{C}$ ). Each sample was divided into two: $5 \mathrm{ml}$ of sample were deposited in an empty glass tube and another $5 \mathrm{ml}$ in a tube containing $1 \mathrm{ml}$ of Iodixanol (Cushion ${ }^{\circledR}$ Fluid). They were centrifuged at $2200 \mathrm{rpm}$ $(800 \mathrm{~g})$ for 20 minutes [11].

After removing them from the centrifuge, the supernatant was aspirated in first place and then the Iodixanol, draining as much as possible without dragging the spermatozoa.

The resulting pellets were resuspended in $5 \mathrm{ml}$ Thilmant medium [11].

The temperature of each sample was gradually lowered during 120

Table 1: Individual motility classification according to Cabodevilla and Catena 2012.

\begin{tabular}{|c|l|}
\hline $\begin{array}{c}\text { Descriptive } \\
\text { value }\end{array}$ & \multicolumn{1}{c|}{ Movement speed } \\
\hline 0 & No movement \\
\hline 1 & Slight ripple or vibration of the tail, no progression \\
\hline 2 & $\begin{array}{l}\text { Slow progression, including stopping and starting the } \\
\text { movement }\end{array}$ \\
\hline 3 & Continuous progressive movement and moderate speed \\
\hline 4 & Rapid progressive movement \\
\hline 5 & $\begin{array}{l}\text { Very fast progressive movement in which the cells are } \\
\text { difficult to follow visually }\end{array}$ \\
\hline
\end{tabular}

minutes until reaching $5^{\circ} \mathrm{C}$, temperature at which they remained 24 hours until their evaluation.

The seminal quality of each sample refrigerated for 24 hours was evaluated in the same way as it had been done for both fresh and frozen semen, studying the same parameters: individual movement quality, percentage of motile spermatozoa, vitality using eosin-nigrosine staining and morphoanomalies.

\section{Results}

The results are shown as the average of the values obtained in each of the 3 groups: Fresh semen (F), semen centrifuged with Iodixanol (I) and semen centrifuged without Iodixanol (NI). Statistical analysis was carried out using Statgraphics ${ }^{\bullet}$ centurion. We analyzed the averages of each parameter using the F-test in the ANOVA table to determine if there are significant differences between the means. Multiple-range tests determined which means were significantly different from others, with a $95 \%$ level of confidence.

The average seed quality of each male used is also shown (Table 2).

The two young males had, on average, worse semen quality than the adults, especially in the parameters of sperm concentration and individual motility. The $\%$ of motile and viable spermatozoa was higher in adult males 1 and 2 compared to the others.

Similar results were obtained for each male, seminal quality was slightly higher for samples treated with Iodixanol (except for the morphoanomaly parameter of young male 1 and adult male 2, which presented higher $\%$ of abnormal forms in samples treated with Iodixanol), without being significantly higher ( $p>0.05$ in all cases).

In order to eliminate the individual effect of the quality of each specimen, the average of each evaluated parameter was obtained, adding the values of all the males, without distinguishing individually between them. The results are shown in the following (Tables 3-7).

The differences are significant between fresh semen samples and chilled semen samples, but they are not significant between I and NI centrifuged samples in both the individual motility parameter and the percentage of motile spermatozoa.

For the parameter $\%$ of viable spermatozoa there are no significant differences between any of the 3 groups studied.

There are significant differences in the average morphoanomalies found between fresh semen and semen centrifuged with Iodixanol, with a greater difference found in fresh semen.

The differences between fresh semen and semen centrifuged without Iodixanol and between the two centrifuged and chilled samples are not significant.

Within the average values of the parameters obtained in semen treated with and without Iodixanol, we found that there are no significant differences between the two, but they are slightly greater in treatment with Iodixanol. The same occurs when maximum values are observed, always favorable for treatment with the padding medium, although these differences do not become significant in the average value of the parameter.

When observing the resulting pellets after centrifugation, we noticed that in the tube without Iodixanol, the spermatozoa pellet appeared strongly adhered to the bottom of the centrifuge tube. This pellet required a strong mechanical treatment for its homogenization in the resuspension medium, and in most cases there were accumulations of sperm cells that did not mix completely. On the other hand, in the seminal fraction that had been centrifuged 
Table 2: Average fresh seminal quality of each male used.

\begin{tabular}{|c|c|c|c|c|c|}
\hline & Young male1 & Young male 2 & Adult male 1 & Adult male 2 & Adult male 3 \\
\hline Volume ejaculate (ml) & 0.5 & 0.7 & 1.3 & 2.5 & 1 \\
\hline Concentration total spermatozoa $\left(\times 10^{6}\right)$ & 1110 & 3610 & 6781 & 8500 & 5533 \\
\hline Individual motility & 3 & 3.85 & 4.07 & 4.12 & 4.08 \\
\hline$\%$ Mobile Sperm & 36 & 57 & 76.43 & 82.5 & 60 \\
\hline Vital stain (\% alive) & 36 & 57.74 & 70 & 71 & 51 \\
\hline Morphoanomalies(\% anomalous) & 41.8 & 45.2 & 46.86 & 40.5 & 27.33 \\
\hline
\end{tabular}

Table 3: Average semen quality of each male after $\mathrm{Cl}$ and $\mathrm{SI}$ refrigeration.

\begin{tabular}{|c|c|c|c|c|c|c|c|c|c|c|}
\hline & \multicolumn{2}{|c|}{ Young male 1} & \multicolumn{2}{|c|}{ Young male 2} & \multicolumn{2}{|c|}{ Adult male 1} & \multicolumn{2}{|c|}{ Adult male 2} & \multicolumn{2}{|c|}{ Adult male 3} \\
\hline & I & NI & I & NI & I & NI & I & NI & I & NI \\
\hline Individual motility & 1.15 & 1 & 2.6 & 1.9 & 2.7 & 2.5 & 2.6 & 2.2 & 3.3 & 2.8 \\
\hline$\%$ mobilesperm & 13 & 10 & 38 & 30 & 37.1 & 36.4 & 50 & 45 & 40 & 40 \\
\hline Vital stain ( $\%$ alive) & 17.8 & 13.1 & 44.3 & 44.6 & 62.4 & 62 & 61.6 & 60.4 & 45.1 & 43.7 \\
\hline $\begin{array}{l}\text { Morphoanomalies } \\
\text { (\% anomalous) }\end{array}$ & 33 & 29.2 & 24.8 & 25.8 & 36.4 & 46.1 & 30.5 & 28.7 & 23.7 & 25.7 \\
\hline
\end{tabular}

Table 4: average of the individual motility parameter, standard deviation and maximum and minimum value obtained in each group.

\begin{tabular}{|c|c|c|c|c|c|}
\hline & $\mathbf{n}$ & Average & Standard Deviation & Minimum & Maximum \\
\hline F & 22 & $3,8^{\text {a }}$ & 0,61 & 2,0 & 4,5 \\
\hline I & 22 & $2,4^{\text {b }}$ & 1,34 & 0 & 3,75 \\
\hline NI & 22 & $2,1^{\text {b }}$ & 1,29 & 0 & 3,5 \\
\hline
\end{tabular}

Different letters denote statistical difference $(p \leq 0,05)$

Table 5: Average of the percentage of mobile spermatozoa obtained in each of the group's evaluated standard deviation and maximum and minimum value.

\begin{tabular}{|c|c|c|c|c|c|}
\hline & $\mathbf{n}$ & Average & Standard Deviation & Minimum & Maximum \\
\hline F & 22 & $61,1^{\text {a }}$ & 19,33 & 30,0 & 90,0 \\
\hline I & 22 & $33,4^{\text {b }}$ & 20,20 & 0 & 60,0 \\
\hline NI & 22 & $30,7^{\text {b }}$ & 19,54 & 0 & 50,0 \\
\hline
\end{tabular}

Different letters denote statistical difference $(p \leq 0,05)$

with Iodixanol, the spermatozoa pellet was observed as a white line between the supernatant and the Iodixanol at the bottom of the tube, and in this case it was easily resuspended in a homogeneous way in the new medium with just a slight agitation, without the need for high mechanical forces, and without the presence of macroscopic cellular accumulations.

\section{Discussion}

One of the major concerns when processing seminal samples by centrifugation is to recover as many sperm as possible with the least possible damage to sperm integrity. In this work we studied whether the centrifugation of goat semen with Iodixanol improved the quality of the semen with respect to those same sperm samples centrifuged without it. Our initial hypothesis focused on the fact that Iodixanol would buffer the mechanical damage that sperm cells sustained during the centrifugation process due to gravitational forces, and therefore
Table 6: Average percentage of viable spermatozoa with eosin-nigrosine vital stain, standard deviation and maximum and minimum value obtained in each group

\begin{tabular}{|c|c|c|c|c|c|}
\hline & $\mathbf{n}$ & Average & Standard Deviation & Minimum & Maximum \\
\hline F & 22 & $57,0^{\text {a }}$ & 16,93 & 22,5 & 86,0 \\
\hline I & 22 & $45,7^{\text {a }}$ & 25,51 & 0 & 85,0 \\
\hline NI & 22 & $44,3^{\text {a }}$ & 26,25 & 0 & 80,0 \\
\hline
\end{tabular}

Different letters denote statistical difference $(p \leq 0,05)$

Table 7: Average Percentage of Sperm Morphoanomalies (Abnormal Forms), Standard Deviation and Maximum and Minimum Value Obtained in Each Group.

\begin{tabular}{|c|c|c|c|c|c|}
\hline & $\mathbf{n}$ & Average & Standard Deviation & Minimum & Maximum \\
\hline F & 22 & $41,6^{\mathrm{a}}$ & 12,33 & 15,0 & 62,0 \\
\hline I & 22 & $30,7^{\mathrm{b}}$ & 16,88 & 7,0 & 68,0 \\
\hline $\mathrm{NI}$ & 22 & $33,3^{\mathrm{a}, \mathrm{b}}$ & 17,84 & 8,0 & 84,0 \\
\hline
\end{tabular}

Different letters denote statistical difference $(p \leq 0,05)$

would improve their viability with respect to samples centrifuged without it. Iodixanol is a product traditionally used in density gradients for cell fractionation [12,13] that has been traditionally used in equine semen washing with good results in recovering the number of undamaged spermatozoa [14-16]. The first work that reported the use of Iodixanol in equine sperm centrifugation was by [17]. They reported that semen centrifuged at $600 \mathrm{~g}$ for 15 minutes without Iodixanol had $30 \%$ less $(\mathrm{P}<0.05)$ live/normal sperm than semen centrifuged at 1000 $\mathrm{g}$ for 25 minutes with Iodixanol [16]. Compared centrifugation at 1000 g for 20 minutes using $5 \mathrm{ml}$ of Cushion-Fluid ${ }^{\circledR}$ with centrifugation at $600 \mathrm{~g}$ for 10 minutes without it. They obtained no significant difference in progressive motility after thawing, but sperm recovery was significantly higher when using the padded centrifugation technique. Other authors $[16,18-20]$ also obtained greater sperm recovery with less apparent sperm damage in equine semen [21,22], using porcine semen, found no significant differences in viability and 
functionality of samples centrifuged with and without Iodixanol, but sperm loss after centrifugation was lower in samples centrifuged using this mattress, concluding that centrifugation with Iodixanol improved the sperm recovery rate without affecting sperm quality. Our results with goat semen did not show significant differences in the assessed seminal quality parameters between centrifuged I and NI samples $(\mathrm{P}>0.05)$, but they were slightly superior on all averages, especially in the percentage of motile spermatozoa, where there is almost a $3 \%$ difference with respect to the NI samples. They gave lower viability results than other authors who worked with chilled goat semen $[23,24]$, due to the low fresh seminal quality of the young males, which decreased the average number of viable spermatozoa. However, the aim of this study was to compare seminal washing with and without Iodixanol, and both in good quality semen and in semen with lower viability and motility rates and lower concentration, the differences in quality were not significant with or without treatment. Where we did find the greatest differences was in the resuspension of the sperm pellet in the incubation medium. The lack of adhesion to the tube in the samples treated with Iodixanol allowed resuspension to be carried out homogeneously and without the use of further mechanical forces such as pipetting, which was required to detach and homogenize the pellets from the samples centrifuged without Iodixanol. Even with the use of pipetting forces, more undissolved spermatozoa were left in NI samples than in I samples. This would mean that sperm recovery would be lower in NI samples, since spermatozoa adhering to the glass or forming clumps would not be recovered. This, together with allowing for higher centrifugation forces and times, as published by the abovementioned authors, would result in a higher number of undamaged recovered spermatozoa, since centrifugation with Iodixanol would avoid causing further mechanical damage to the sperm cells in order to achieve their resuspension.

\section{Conclusions}

Centrifugation of caprine seminal samples with Iodixanol does not affect the quality of the samples kept in refrigeration at $5^{\circ} \mathrm{C}$, but it is useful to facilitate sperm homogenization in the resuspension medium, which would result in an increase in the number of undamaged recovered spermatozoa. This increased rate of goat sperm recovery needs to be confirmed in future studies.

Treatment with Iodixanol does not affect the quality of spermatozoa in young goats or adults, irrespective of the starting sperm quality of the animals.

Nor does it depend on the dilution of the spermatozoa before or after centrifugation.

\section{References}

1. Aamdal J, Lyngset O, Fossum K (1965) Toxic effect of lysolecithin on sperm. Nord Vet Med 17: 633-639.

2. Iritani A, Nishikawa Y (1963) Studies on the egg yolk coagulating enzyme in goat semen IV. On the position of yolk constituents attacked by the coagulating enzyme. Jpn J Anim Reprod 8: 113-117.

3. Carvajal G, Cuello C, Ruiz M, Vázquez JM, Martínez EA, et al. (2004) Effects of centrifugation before freezing on boar sperm cryosurvival. J Androl 25: 389-396.

4. Aitken RJ, Clarkson JS (1988) Significance of reactive oxygen species and antioxidants in defining the efficacy of sperm preparation techniques. J Androl 9: 367-376.

5. Mortimer D (1991) Sperm preparation techniques and iatrogenic failures of in-vitro fertilization. Hum Reprod 6: 173-176.
6. Alvarez JG, Lasso JL, Blasco L, Nuñez RC, Heyner S, et al. (1993) Centrifugation of human spermatozoa induces sublethal damage; separation of human spermatozoa from seminal plasma by a dextran swim-up procedure without centrifugation extends their motile lifetime. Hum Reprod 8: 1087-1092.

7. Waite, Jessica Arlene (2007) Cushioned centrifugation of stallion semen: factors impacting equine sperm recovery rate and Quality. Texas A\&M University.

8. Corteel JM, Baril G (1974) Viabilité des spermatozoïdes de bouc conservés et congelés avec ou sans leur plasma séminal : effet du glucose. Ann Biol anim Bioch Biophys 14: 741-745.

9. Cabodevila J, Catena M (2012) Evaluación de semen bovino congelado. Engormix.

10. Colas G, Guerin Y (1980) Variations saisonnières de la qualité du sperme chez le bélier lle-de-France. I. Etude de la morphologie cellulaire et de la motilité massale. Reprod Nutr Dev 20: 1789-1799.

11. Thilmant $P$ (1997) Congelation du sperme de verrat en poillete de 0,5 ml. Resultats sur le terrain. Ann Med Vet 141: 457-462.

12. Bendixen B, Rickwood D (1994) Properties and applications of a new type of iodinated gradient medium. J Biochem Biophys Methods 28: 43-52.

13. Van Veldhoven PP, Baumgart E, Mannaerts GP (1996) lodixanol (Optiprep), an improved density gradient medium for the isoosmotic isolation of rat liver peroxisomes. Anal Biochem 237: 17-23.

14. Ecot P, Decuadro-Hansen G, Delhomme G, Vidament M (2005) Evaluation of a cushioned centrifugation technique for processing equine semen for freezing. Anim Reprod Sci 89: 245-248.

15. Knop K, Rath D, Sieme H (2005) Effects of duration, force of centrifugation and cushioned centrifugation technique on sperm recovery and sperm quality in stallions with good and poor semen freezability. Havemeyer Foundation Mono Series 18: 6-9.

16. Knop K, Hoffmann N, Rath D, Sieme H (2005) Effects of cushioned centrifugation technique on sperm recovery and sperm quality in stallions with good and poor semen freezability. Anim Reprod Sci 89: 294-297.

17. Revell SG, Pettit MT, Ford TC (1997) Use of centrifugation over iodixanol to reduce damage when processing stallion sperm for freezing. Proc Joint Meeting, Society for the Study of Fertility, Abstr Series No 92: 38.

18. Loomis PR (2006) Advanced methods for handling and preparation of stallion semen. Vet Clin North Am Equine Pract 22: 663-676.

19. Waite JA, Love CC, Brinsko SP, Teague SR, Salazar JL Jr, et al. (2008) Factors impacting equine sperm recovery rate and quality following cushioned centrifugation. Theriogenology 70: 704-714.

20. Hoogewijs M, Rijsselaere T, De Vliegher S, Vanhaesebrouck E, De Schauwer C (2010) Influence of different centrifugation protocols on equine semen preservation. Theriogenology 74: 118-126.

21. Gadea J, Martínez-Miró S, Decuadro-Hansen G, Matás C (2005) Evaluation of Boar Sperm Functionality after A Cushioned Centrifugation Technique. Reprod Fert Develop 18: 154.

22. Matás C, Decuadro G, Martínez-Miró S, Gadea J (2007) Evaluation of a cushioned method for centrifugation and processing for freezing boar semen. Theriogenology 67: 1087-1091.

23. Martínez J, Hernández JL, Díaz N, Molina Y, Interian L, et al. (2007) un nuevo diluyente para la conservación del semen caprino en estado fresco refrigerado. Ciencia y Tecnología. Ganadera 1: 127-132.

24. Palomino JM, Cervantes M, Rodríguez A, Cisneros F, Huanca W (2007) Efecto de dos dilutores y tiempos de refrigeración sobre la motilidad individual de semen refrigerado de caprinos. Sitio Argentino de Producción Animal. 